\title{
A Scaling Model for Predicting the Gas-Channel Formation Period in Crater-Like Electrospinning of Nanofibers
}

\author{
Jian Liu', Wan Shou' ${ }^{2}$ Lei Zhang ${ }^{2}$, Wei Liang², Dong-Wei Huang ${ }^{3}$ and Yong Liu ${ }^{* 2}$ \\ ${ }^{I}$ Department of Textiles, Zhejiang Fashion Institute of Technology, No.495 Fenghua Road, Ningbo, Zhejiang Province, \\ 31521, P.R. China \\ ${ }^{2}$ Key Laboratory of Advanced Textile Composites, Ministry of Education of China, School of Textiles, Tianjin \\ Polytechnic University, No.399 West Binshui Road, Tianjin 300387, P.R. China \\ ${ }^{3}$ School of Science, Tianjin Polytechnic University, No.399 West Binshui Road, Tianjin 300387, P.R. China
}

\begin{abstract}
Crater-like electrospinning is a novel and cost-effective method for the mass scale production of nanofibers. The gas channel in the polymer solution plays a key role to produce a bubble Taylor cone or a crater-like Taylor cone, which is the key to eject the thin fluid jets (finally solidified into nanofibers) in electrospinning process. However, the formation mechanism of gas channel of crater-like Taylor cone is still unclear, which hinders further development of this process. In this work, a simple and effective scaling model was firstly established to predict the period of the gas-channel formation in the polymer solution during electrospinning process. Our theoretical analysis showed that the gas-channel formation period was mainly determined by the input air pressure during the process. The relationship between the formation period and the input air pressure followed a scaling law. In order to verify the model, crater-like electrospinning process was carried out and a high-speed digital camera was employed to observe the gas channel. The experimental results agree well with the scaling model, which indicates that the proposed system is feasible. The scaling model could be useful in helping us to understand the process.
\end{abstract}

Keywords: Electrospinning, mathematics analysis, nanofibers, polymer, scaling model.

\section{INTRODUCTION}

In the 1930s electrospinning was developed as one of the important methods for fiber fabrication, and has produced various polymer nanofibers with the diameter range from several nanometers to several micrometers [1-3]. The nanofibrous materials obtained by electrospinning has nonwoven structure with unique features, including interconnected pores and a very large surface to volume ratio, which enable such nanofiber materials to have many applications such as filters, energy storage, environment engineering, protective clothing and tissue engineering $[4,5]$. With the rapid increase in application of nanofibers in more and more industry areas, electrospinning has been one of the most important and basic techniques in the fabrication of nanofibers since the early 2000 s.

These applications require large number of electrospun fibers, however, the major challenges encountered in the electrospinning process are poor efficiency and low output, about $0.01-0.3 \mathrm{~g} / \mathrm{h}$ [6], which has been the technological bottleneck for the industry. Many researchers has proved that the number of fluid jets (finally turned into nanofibers) was the key to improving the yield of nanofibers. In order to address the low number of jets, some novel electrospinning processes have been invented to produce simultaneously

*Address correspondence to this author at the School of Textiles, Tianjin Polytechnic University, No.399 West Binshui Road, Tianjin 300387, P.R. China; Tel: 13780057669; E-mail: liuyong@tjpu.edu.cn multiple fluid jets and fabricate a high yield of nanofibers recently [7].

Bubble electrospinning and crater-like electrospinning are both new electrospinning techniques having the potential to fabricate nanofibers with a high output, which designed by our group [8-10]. It is well known that the Taylor cone plays a key role in the production of nanofibers in electrospinning process, bubble electrospinning and crater-like electrospinning are no exceptions. In these two processes, Taylor cone, a bubble or a crater-like solution ridge, has significant influence on producing multiple jets continuously. However, bubble electrospinning process is discontinuous due to the repeat of burst and reformation of fluid bubble, according to our previous study [11].

Recently, many researchers have studied bubble electrospinning process and wondered about how to improve the properties and the yield of nanofibers. In order to improve the discontinuity of bubble electrospinning process, the time of gas channel formation should be decreased. Obviously, if a bubble is changed into a crater-like solution ridge, the gas-channel formation period could be shorter, the number of fluid jets could be greater and the yield of nanofibers be higher. This is why a crater-like electrospinning process was developed in our group.

Most previous studies have given the readers basic knowledge for understanding the process [12-14]. Unfortunately, almost all the previous literatures paid their attention on the fabrication of nanofibers. The reason of the 
gas-channel formation in this new electrospinning process has not been investigated. Recently, the mathematical model has become an efficiency tool for solving practical problems in materials science [15]. In this work, the influence of air pressure on the period of gas-channel formation was studied to understand the crater-like electrospinning process.

\section{THEORETICAL ANALYSIS}

Generally, only fluid bubble would be formed on the liquid surface when the viscosity of polymer solution is low. The gas channel was not continuous, as shown in Fig. (1a). When the solution viscosity is high enough and the air pressure is appropriate, the bubble would be transformed into crater-like solution ridge. In this case, the gas channel was formed continuously, as shown in Fig. (1b). If the properties of a polymer solution were fixed, the transformation of Taylor cone from bubble to crater-like elevation would be depended on the air pressure released into the solution.

In order to simply the physical model, some assumptions were given as follows: (1) different air pressure is corresponding to different gas-channel formation period at other conditions fixed, and the same air pressure is corresponding to the same gas-channel formation period at other conditions fixed; (2) the kinetic energy of the system is the same at the same period. (3) the kinetic energy of pressured air in a period is equal to the kinetic energy of liquid plus the potential energy of the crater-like solution elevation.
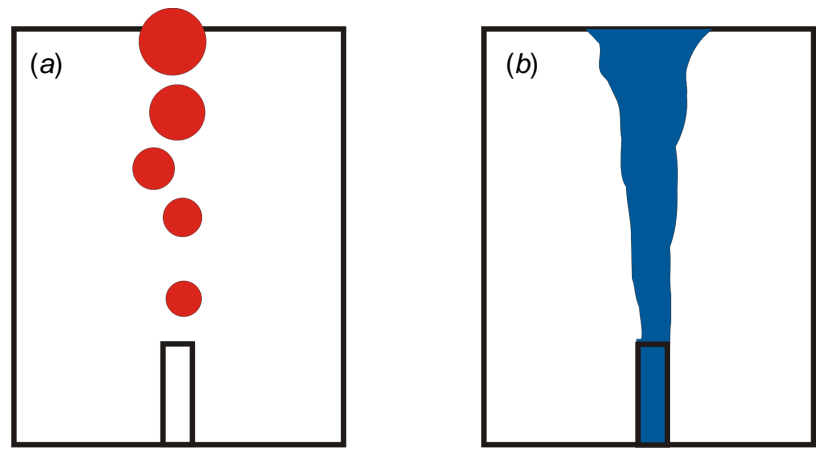

Fig. (1). Gas channel in bubble electrospinning and crater-like electrospinning.

In order to form the crater-like solution ridge on free liquid surface, pressured air must provide enough energy to expel the solution. The energy in a period can be considered to meet the conditions of forming Taylor cone from bubble to crater-like solution elevation. The energy $\mathrm{E}$ in a period can be expressed as

$$
E=1 / 2 m u^{2}
$$

where $m$ is the mass of pressured air, $u$ is the velocity of pressured air.

In crater-like electrospinning, the density of pressured air can be denoted as $\rho_{g}$, the radius of gas tube is $d_{0}$, Eq. (1) can be changed as

$$
E=1 / 2 \pi d_{0}^{2} \rho_{g} T \cdot u^{3}
$$

where $T$ is the gas-channel formation period.

According to above assumptions, $d_{0}, \rho_{g}$ and $E$ can be considered as a constant. So we can obtain

$T=C / u^{3}$

where $C$ is a constant.

According to many empirical observations, the velocity of pressured air is proportional to the air pressure gauge reading $P$, i.e.,

$u \propto P$

Thus Eq. (3) can be expressed as

$T \propto k / P^{3}$

where $k$ is a constant.

Eq. (5) showed that the relationship between air pressure and the period followed scaling laws.

\section{EXPERIMENTAL VERIFICATION}

\subsection{Materials}

Polyvinylpyrrolidone (PVP k-30, $\bar{M}_{w}=30,000-40,000$ $\mathrm{g} / \mathrm{mol}$ ) was purchased from Sinopharm Chemical Reagent Co., Ltd., (Shanghai, China). The solvent, distilled water, was obtained from Tianjin Polytechnic University. All the chemicals were used as received without further purification. The polymer, PVP, was dissolved into distilled water, and the concentration was adjusted to $40 \mathrm{wt} \%$. The mixture was stirred with mechanical stirrer for 2 hours at room temperature. Then the prepared PVP solution was placed into a solution reservoir. A bubble was first produced on the free surface of the polymer solution when the gas pump was turned on slowly. With the increase of air pressure, the bubble was changed to crater-like solution ridge, which was considered as a Taylor cone in electrospinning process.

\subsection{Setup}

The schematic drawing of crater-like electrospinning is shown in Fig. (2a). The crater-like electrospinning process was carried out with an apparatus consisting of a glass cup, a gas pump, a DC high voltage supplier and a grounded collector. A glass gas tube was fixed on the center of the cup bottom. A metal needle, as the positive electrode, went through the tube. The tube was connected with the gas pump. The collector, a flat aluminium plate, was over the cup. The distance between the liquid surface and the grounded electrode could be adjusted. The principles of both electrospinning processes are deceptively simple: in an electric field, a fluid bubble or a crater-like solution elevation, as a Taylor cone in electrospinning, was produced on the liquid surface. Multiple fluid jets were ejected simultaneously from Taylor cone when the applied voltage exceeded the threshold value and finally became nanofibers on the collector.

In this new process, a gas channel, which has a very short period, is formed in the solution. At the same time, multiple jets were ejected from the crater-like Taylor cone, as shown 

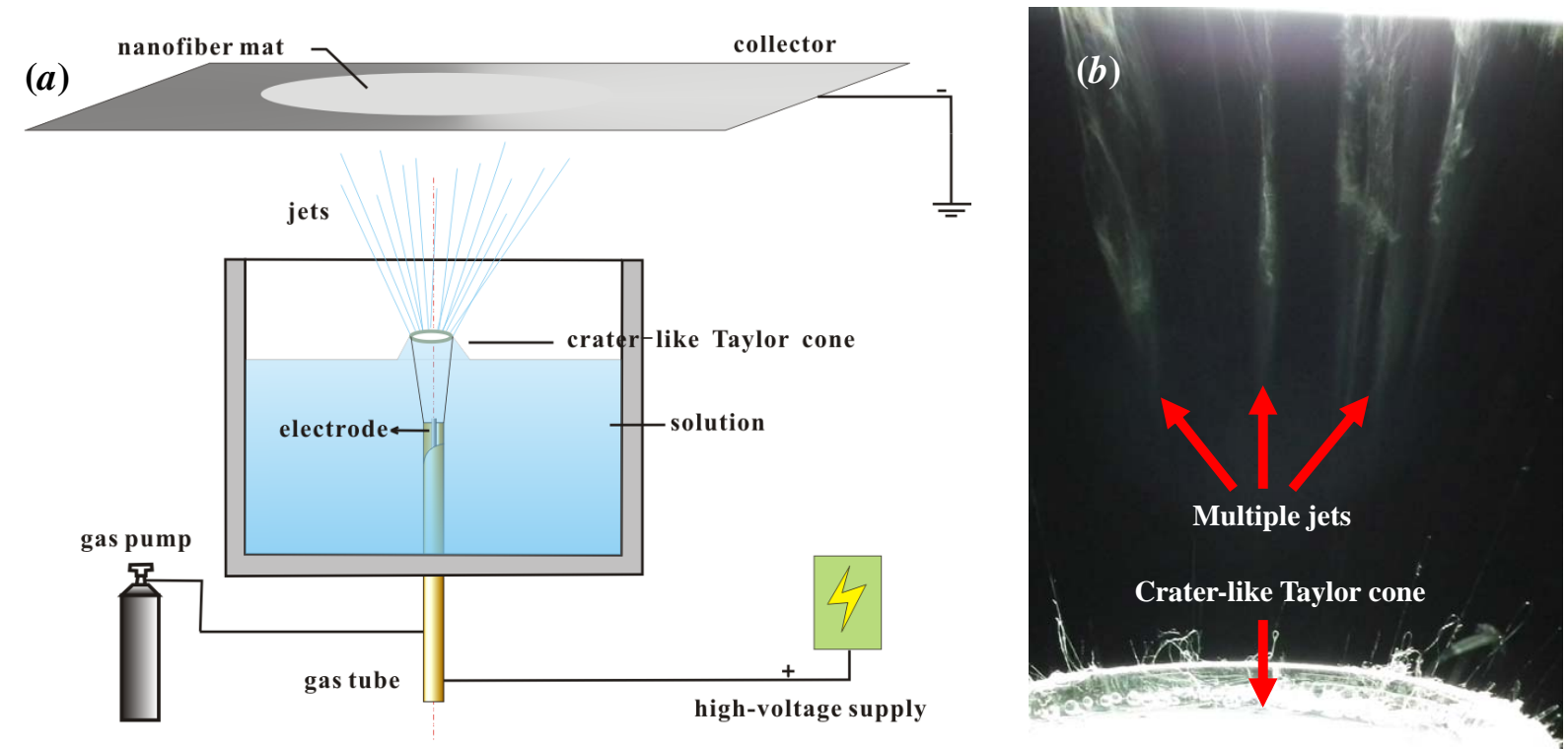

Fig. (2). (a) Schematic drawing of crater-like electrospinning setup. (b) Photograph of crater-like electrospinning process.

in Fig. (2b), which is different with the traditional electrospinning process. According our observations, the formation of Taylor cone, either a bubble or a crater-like solution elevation, on the liquid surface was largely depended upon the released air pressure at other conditions fixed in this electrospinning processes. It is the gas channel formation period that plays an important role in improving the yield of fluid jets.

\subsection{Characterization}

The crater-like electrospinning process was captured by a digital camera (FinePix HS11, FUJIFILM, Japan) with a lens that had lens focal length $\mathrm{f}=4.2-126.0 \mathrm{~mm}$ and $\mathrm{F}$ number of 2.8 (Wide) - 5.6 (Telephoto). The images were produced at 120 frames per second in natural light in this work. The morphologies of the nanofibers were examined by scanning electron microscopy (SEM) (TM-1000, Hitachi, Japan).

\section{RESULTS AND DISCUSSION}

The obtained nanofibers was collected on a collector and then was observed by a scanning electron microscopy, as shown in Fig. (3). The morphologies of nanofibers was typical nonwoven structure with interconnected pores. The diameter of nanofibers ranged from $70 \mathrm{~nm}$ to $1000 \mathrm{~nm}$, the average diameter was $600 \mathrm{~nm}$.

In order to verify the above theoretical analysis, experiments were carried out under the other fixed conditions (solution concentration, solution depth between the liquid surface and the tip of gas tube, etc.). In these experiments, the effect of air pressure on the gas tunnel were studied in the range of $4-50 \mathrm{kPa}$, while all other processing parameters were kept constant (applied voltage $=0 \mathrm{kV}$, solution concentration $=40 \mathrm{wt} \%$, solution depth $=0.8 \mathrm{~cm}$ ).

The high-speed photos of gas channel at different air pressure in these experiments are showed in Fig. $(\mathbf{3 b}, \mathbf{c})$. The air pressure were used as $4 \mathrm{kPa}, 10 \mathrm{kPa}, 16 \mathrm{kPa}, 25 \mathrm{kPa}, 35 \mathrm{kPa}$ and $50 \mathrm{kPa}$, respectively, in our experiments. From these photographs, it could be founded that the period of gaschannel formation decreased with the increase of air pressure in the crater-like electrospinning process. The period was $225 \mathrm{~ms}$ when the air pressure was $4 \mathrm{kPa}$. In this case, only fluid bubble was produced on the liquid surface during the process, which showed that the low air pressure could not produce a crater-like Taylor cone. With the increase of air pressure, the gas-channel formation period decreased obviously. When the air pressure reached $10 \mathrm{kPa}$, the period was $66.7 \mathrm{~ms}$ and a crater-like solution ridge was produced on the liquid surface, as shown in Fig. (3b). The results showed that the air pressure, $10 \mathrm{kPa}$, was a critical air pressure under this condition. However, the crater-like solution ridge was not stable. When the air pressure reached $16 \mathrm{kPa}$, the period was $58.3 \mathrm{~ms}$ and a stable crater-like solution ridge was produced on the liquid surface. The formation period was shorter, e.g., $50 \mathrm{~ms}$, when the air pressure reached $25 \mathrm{kPa}$, see Fig. (3c). It was shown that the period reduced and the crater-like Taylor cone was stable when the air pressure were over $25 \mathrm{kPa}$.

The air pressures and its corresponding periods were listed in Table 1. The experimental results showed that as the gas-channel formation period kept decreasing as the air pressure increased, the fluid jets were produced quickly.

In order to confirm the period dependence on the air pressure, the period was plotted against the air pressure at fixed other processing variables, as shown in Fig. (4). The Equation (6) is the fitting equation of the experimental data (see Fig. 4).

$T=11.45^{*} P^{-3}+0.0468$

where $k=11.45$ in this experiments.

The results showed the experimental data agreed well with the theoretical analysis, which indicates that the proposed scale model is feasible. 

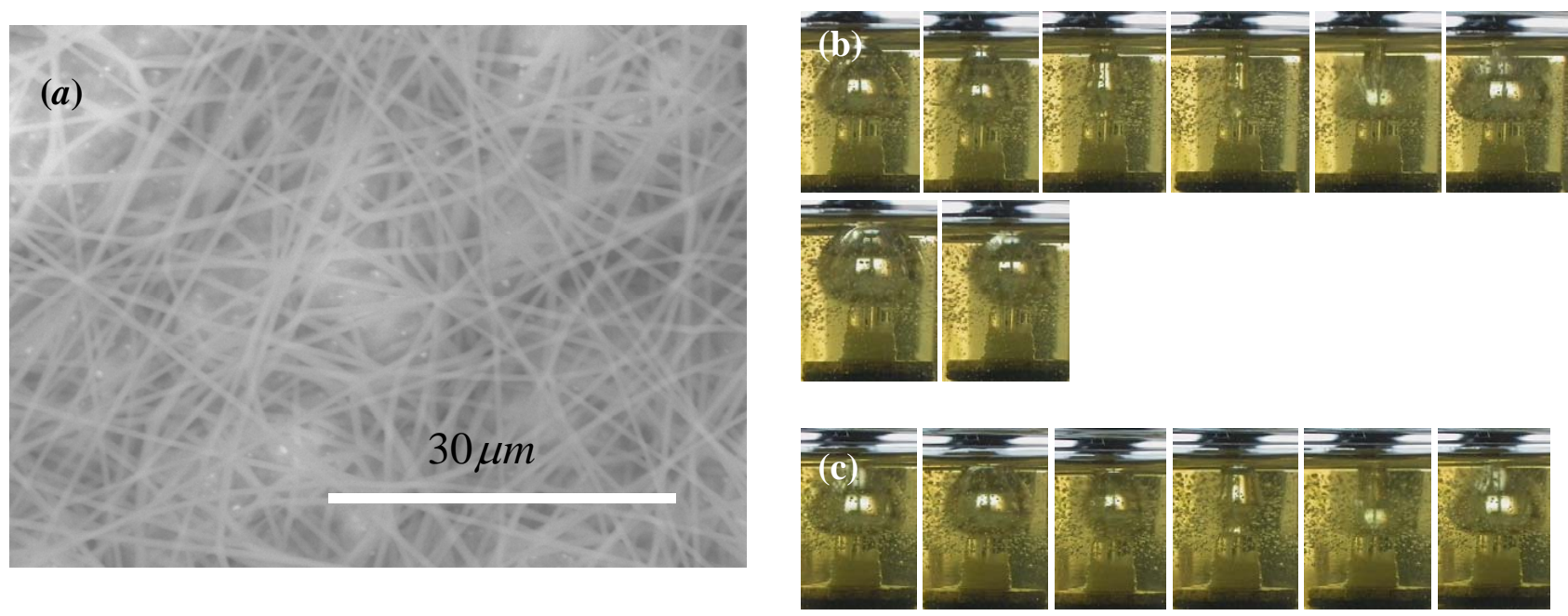

Fig. (3). (a) SEM photo of nanofibers obtained in crater-like electrospinning, (b, c) High-speed photographs of gas channel at different air pressure in crater-like electrospinning process $(\mathbf{b}$ - Air pressure $\mathrm{P}=10 \mathrm{kPa}$, period $\mathrm{T}=66.7 \mathrm{~ms}$; $\mathbf{c}$ - Air pressure $\mathrm{P}=25 \mathrm{kPa}$, period $\mathrm{T}=50 \mathrm{~ms}$ ).

Table 1. The air pressure and its formation period in experiments.

\begin{tabular}{|c|c|}
\hline Air Pressure (kPa) & Period (ms) \\
\hline \hline 4 & 225 \\
\hline 10 & 66.7 \\
\hline 16 & 58.3 \\
\hline 25 & 50.0 \\
\hline 35 & 41.7 \\
\hline 50 & 33.3 \\
\hline
\end{tabular}

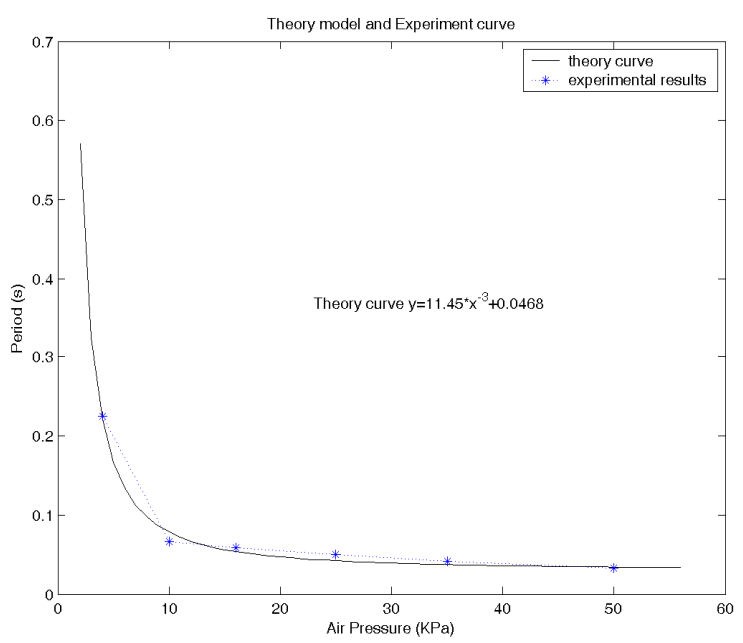

Fig. (4). Theoretical and experimental results of relationship between the air pressure and the period of gas-channel formation.

\section{CONCLUSION}

In this presentation, PVP was used to study the effect of air pressure on gas-channel formation period in crater-like electrospinning process. The theoretical analysis showed that the gas-channel formation period was greatly influenced by air pressure, and the period had an allometric relationship with air pressure. The experimental results verified the above theoretical prediction well. The relationship between the period and the air pressure is in the form, $T \propto P^{-3 / 2}$, in crater-like electrospinning. The scale model might be used to understand the new electrospinning process of nanofibers with great yield.

\section{CONFLICT OF INTEREST}

The author confirms that this article content has no conflict of interest.

\section{ACKNOWLEDGEMENTS}

The present work is supported by Ningbo Natural Science Foundation (No.2013A610016), Project from Zhejiang Fashion Institute of Technology (No. 2013-113002), National Natural Science Foundation of China under Grant (No.51003073), Foundation for the Author of National Excellent Doctoral Dissertation of PR China under Grant (No. 201255), Program for New Century Excellent Talents in University (NCET-12-1063), Tianjin Natural Science Foundation (14JCYBJC17600) and Foundation for Training Program of Young and Middle-aged Innovative Talents in Higher Education of Tianjin, China.

\section{REFERENCES}

[1] T. Han, D. H. Reneker, and A. L. Yarin, "Buckling of jets in electrospinning," Polymer, vol. 48, pp. 6064-6076, 2007.

[2] Y. Liu, J. H. He, J. Y. Yu, H. Zeng, "Controlling numbers and sizes of beads in electrospun nanofibers," Polymer Int., vol. 57, pp. 632636, 2008.

[3] J. Lin, F. Tian, Y. Shang, F. Wang, B. Ding, J. Yu, "Facile control of intra-fiber porosity and inter-fiber voids in electrospun fibers for selective adsorption," Nanoscale, vol. 4, pp. 5136-5120, 2012.

[4] X. Qin, H. Wang, and S. Wu, "Investigation on structure and thermal properties of electrospun cellulose diacetate nanofibers," $J$. Indust. Textiles, vol. 42, pp. 244-255, 2012. 
[5] J. Y. Lin, F. Tian, Y. Shang, F. Wang, B. Ding, J. Yu, Z. Guo, “Coaxial electrospun polystyrene/polyurethane fibres for oil collection from water surface," Nanoscale, vol. 5, pp. 2745-2755, 2013.

[6] H. Niu, X. Wang, and T. Lin, "Needleless electrospinning: influences of fibre generator geometry," J. Textile Inst., vol. 103, pp. 787-794, 2012.

[7] P.-C. Li, Y.-B. Liu, and W.-Y. Chen, "Research on sawtooth type of needleless electrostatic spinning," J. Mod. Textile Sci. Eng., vol. 2, pp. 25-34, 2011.

[8] Y. Liu, and J.-H. He, "bubble electrospinning for mass production of nanofibers," Int. J. Nonlinear Sci. Numer. Simulat., vol. 8, pp. 393-396, 2007.

[9] J.-H. He, and Y. Liu, "Control of bubble size and bubble number in bubble electrospinning," Comput. Math. Appl., vol. 64, pp. 10331035, 2012.

[10] Y. Y. Wang, Y. Liu, W. Lian, M. Ma, R. Wang, "Fabrication of nanofibers via Crater-like electrospinning," Adv. Mat. Res., vol. 332-334, pp. 1257-1260, 2011.
[11] Y. Liu, L. Dong, J. Fan, R. Wang, J. Yu, "Effect of applied voltage on diameter and morphology of ultrafine fibers in bubble electrospinning," J. Appl. Polym. Sci., vol. 120, pp. 592-598, 2011.

[12] Y. Liu, W. Liang, W. Shou, Y. Su, R. Wang, "Effect of temperature on the crater-like electrospinning process," Heat Transfer Res., vol. 44, pp. 447-454, 2013.

[13] Y. Liu,. J. Li, Y. Tian, X. Yu, J. Liu, and B.M. Zhou, "CLSVOF method to study the formation process of taylor cone in crater-like electrospinning of nanofibers," J. Nanomat., vol. 2014, Article ID 635609, 12 pages, 2014.

[14] Y. Liu, J. Li, Y. Tian, J. Liu, and J. Fan, "Multi-physics Coupled FEM method to simulate the formation of crater-like taylor cone in electrospinning of nanofibers," J. Nano Res., vol. 27, pp. 153-162, 2014.

[15] J. H. He, Y. Q. Wan, and J. Y. Yu, "Scaling law in electrospinning: relationship between electric current and solution flow rate," Polymer, vol. 46, pp. 2799-2801, 2005.

Received: September 24, 2014

Revised: September 30, 2014

Accepted: October 22, 2014

(C) Liu et al.; Licensee Bentham Open.

This is an open access article licensed under the terms of the Creative Commons Attribution Non-Commercial License (http://creativecommons.org/licenses/ by-nc/3.0/) which permits unrestricted, non-commercial use, distribution and reproduction in any medium, provided the work is properly cited. 